\title{
Discovery of a RuBisCO-like Protein that Functions as an Oxygenase in the Novel D-Hamamelose Pathway
}

\author{
Suk Min Kim, Hyun Seung Lim, and Sun Bok Lee
}

(C) The Korean Society for Biotechnology and Bioengineering and Springer 2018

In the 2018 issue of Biotechnology and Bioprocess Engineering (BBE), an error occurred in the research article: Suk Min Kim, Hyun Seung Lim, and Sun Bok Lee (2018) Discovery of a RuBisCO-like Protein that Functions as an Oxygenase in the Novel D-Hamamelose Pathway. Biotechnol. Bioprocess Eng. 23: 490-499. In Fig. 3, L-Lyxose and LLyxonate should be replaced by D-Ribose and D-Ribonate, respectively. A revised Fig. 3 is presented here.

A<smiles>[R][C@H]1O[C@H](O)[C@]([R2])(O)[C@H]1O</smiles>

- D-Hamamelose: $\mathrm{R}_{1}=\mathrm{R}_{2}=\mathrm{CH}_{2} \mathrm{OH}$

-D-Erythrose: $\mathbf{R}_{1}=\mathbf{R}_{2}=\mathrm{H}$

- D-Ribose: $\mathrm{R}_{1}=\mathrm{CH}_{2} \mathrm{OH}, \mathrm{R}_{2}=\mathrm{H}$
B

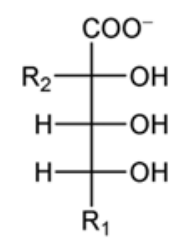

\footnotetext{
-D-Hamamelonate: $\mathrm{R}_{1}=\mathrm{R}_{2}=\mathrm{CH}_{2} \mathrm{OH}$ - D-Erythronate: $\mathrm{R}_{1}=\mathrm{R}_{2}=\mathrm{H}$ -D-Ribonate: $\mathrm{R}_{1}=\mathrm{CH}_{2} \mathrm{OH}, \mathrm{R}_{2}=\mathrm{H}$
}

Fig. 3. (A) Structural similarities between D-hamamelose and other aldoses. (B) Structural similarities between D-hamamelonate and other aldonates.

Suk Min Kim ${ }^{\dagger}$, Hyun Seung Lim ${ }^{\dagger}$, Sun Bok Lee

Department of Chemical Engineering, Pohang University of Science and Technology, Pohang, Korea

Tel: +82-54-279-2268; Fax: +82-54-279-2699

E-mail:sblee@postech.ac.kr

'Both authors contributed equally to this work. 